\title{
Association of Multidrug Resistance Gene-1 (MDR1 C1236T) Polymorphism with the Risk of Acute Myeloid Leukemia in a Moroccan Population
}

\author{
Oum Kaltoum Ait Boujmia ${ }^{1,2 *}$, Sellama Nadifi ${ }^{1}$, Hind Dehbi ${ }^{1,2}$, Mouna \\ Lamchahab $^{3}$, Asma Quessar ${ }^{3}$
}

\begin{abstract}
The human multidrug resistance $M D R 1$ gene plays a crucial role in the absorption, transport, metabolism and elimination of harmful compounds. An impaired metabolism of these compounds related to genetic polymorphism may cause cancer such as acute myeloid leukemia AML. Objective: The present study investigated the relationship between C1236T polymorphism and the risk of AML development in a sample of Moroccan population. Methods: The present case-control study included 131 AML patients and 136 healthy controls. The MDR1 C1236T polymorphism was identified by PCR-RFLP method. Meta-analysis was performed to discuss our results. Statistical analyses were performed using SPSS, MetaGenyo and MedCalc. Results: A positive association was found between the 1236TT mutant genotype and the risk of AML (OR 2.39; 95\% CI 1.02-5.57, p=0.04) compared to the wild type 1236CC. In addition, the recessive model revealed that carriers of 1236TT mutant genotype were more exposed to develop AML when compared to the combined 1236CC/CT genotype (OR: 2.27, CI: 1.01-5.05, $\mathrm{p}=0.04$ ). The clinical parameters of AML showed no significant association. Meta-analysis demonstrated no statistically significant association between this polymorphism and AML susceptibility. Conclusion: Our study suggests that the MDR1C1236T polymorphism appears to be associated with the risk of AML. Further studies, including a large sample size, are needed to confirm these findings.
\end{abstract}

Keywords: $P$-gp-MDRl- acute myeloid leukemia- single nucleotide polymorphism- $C 1236 T$ - Meta-analysis

Asian Pac J Cancer Prev, 21 (7), 1899-1904

\section{Introduction}

Acute myeloid leukemia (AML) is the most deadly type of leukemia (GB, 2016). As other types of cancer, the etiology of AML remains unknown. However, many risk factors were incriminated such as exposition to pesticides, radiotherapy, chemotherapy, benzene and radiation (Zeeb and Blettne, 1998; Smith et al., 2011). In addition, many genes have been reported to be associated with higher risk of AML.

$M D R 1$ or $A B C B 1$ gene is expressed in organs implicated in xenobiotics metabolism and excretion (the gastrointestinal system, kidney and liver). Therefore, $M D R 1$ plays a crucial role in the elimination of carcinogenic substances. The alteration of $M D R 1$ activity related to interindividual genetics background may lead to the development of cancers (Laura et al., 2011; Urayama et al., 2007; Ambudkay et al., 1999; Schimkel, 1997; Hattori et al., 2007).

In humans, the MDRl gene is located on chromosome 7, at position $\mathrm{q} 21$, which encodes for a transmembrane protein that plays role of ATP -dependent efflux transporter pump, called P-glycoprotein (P-gp) (Clarke et al., 2005). MDRl is a highly polymorphic gene. About a thousand single nucleotide polymorphisms (SNPs) have been found (Bodor et al., 2005; Hodges et al., 2011).

The synonymous SNP C1236T in exon12 (rs1128503) is one of the most studied SNPs in this gene, that leads to the alteration of MDR1 expression and activity (Marzolini etal., 2004). The allele frequencies of this polymorphism vary from $30 \%$ to $93 \%$ between different ethnic groups worldwide (Hodges et al., 2011) Several genetic epidemiological studies reported a positive association between this polymorphism in MDR1 gene and cancer risk such us, acute lymphoblastic leukemia, breast cancer, acute myeloid leukemia and non-hodgkin lymphoma (Talaat et al., 2018; Abuhaliema et al., 2016; Ait Boujmia et al., 2020; Kim et al., 2014)

The purpose of the present study was to explore the relationship between C1236T polymorphism and AML

${ }^{1}$ Laboratory of Cellular and Molecular Pathology, Faculty of Medicine and Pharmacy of Casablanca, University Hassan II, Casablanca, Moroccoa. ${ }^{2}$ Morocco Laboratory of medical Genetics, CHU Ibn Rochd, Casablanca, Morocco. ${ }^{3}$ Department of Onco-Hematology, Ibn Rochd University Hospital, Casablanca, Morocco. *For Correspondence: kaltoum.biologie@gmail.com 
susceptibility.

\section{Materials and Methods}

Patients and Methods

Study population

The present case-control study included 131 AML patients and 136 healthy age-matched unrelated controls, recruited from the Department of Hematology and Pediatric Oncology, 20 August Hospital, University Hospital, Ibn Rochd, Casablanca Morocco from 2015 to 2017. Individual written informed consent was obtained from all participants. The classification of AML subtypes in patients was performed according to the criteria of the World Health Organization 2008 (WHO) (Vardiman et al., 2009).

\section{Genotyping of C1236T}

Genomic DNA was extracted by the salting-out protocol (Miller et al., 1998). DNA concentration was estimated by NanoVue Plus spectrophotometer. Genotypic identification of the C1236T MDR1 polymorphism was performed using polymerase chain reaction followed by the restriction fragment length polymorphism (PCR-RFLP) technique previously described by Kassogue et al., (2014). The PCR reaction was carried out in a final volume of $25 \mu \mathrm{l}$. The PCR products were digested overnight with HaeIII and analyzed on a $3 \%$ agarose gel stained with ethidium bromide.

\section{Meta-Analysis \\ Retrieval strategy}

Literature search was accomplished independently by two investigators to find all articles published before March 27, 2019 that investigating the relationship between the polymorphisms and AML risk, using the following key words: P-gp, multidrug resistance gene, $M D R 1, A B C B 1$, acute myeloid leukemia, AML, polymorphism, C1236T, SNP. Eligible studies were found by searching PubMed, Science Direct and Google Scholar.

\section{Inclusion and exclusion criteria}

The inclusion criteria were: studies about the association between the MDR1 C1236T polymorphism and AML with sufficient data were included. The exclusion criteria were: chronic myeloid leukemia, acute lymphoid leukemia (CML, ALL) and lymphoma studies, review articles, studies that did not meet our study and duplicate studies.

\section{Pooled Studies for the Meta-Analysis}

Four case-control studies investigating MDR1 C1236T polymorphism in AML patients were included in the present meta-analysis with 480 cases and 920 controls Figure 1.

\section{Statistical analysis}

Statistical analyzes were performed using SPSS (version 16; SPSS Inc., Chicago, IL, USA) and MedCalc (version 8; MedCalc Software, Mariakerke, Belgium) as well as MetaGeny (GENYO). The Hardy-Weinberg equilibrium was performed by chi-square test in patients and controls. The odds ratio (OR) with $95 \%$ confidence intervals $(\mathrm{CI})$ was calculated to assess the association between the C1236T MDR1 polymorphism and AML risk. We used either the chi-square or Fisher's exact tests to evaluate the association between C1236T MDR1 polymorphism and clinical parameters; a p value less than 0.05 was considered statistically significant. The dominant genetic model was defined as TT homozygote + CT heterozygote mutant genotypes compared with homozygote Wild-type genotype $\mathrm{CC}$, while the recessive genetic model was defined as TT homozygote mutant compared with heterozygote+ homozygote Wild-type genotypes. For the meta-analysis the ORs were estimated using a fixed-effects model. Genetic Heterogeneity was estimated with the Cochran's $Q$ test and $\mathrm{I}^{2}$ statistics was used to estimate the heterogeneity among studies. A random-effects model was used to estimate common OR if heterogeneity was detected; $\mathrm{I}^{2}>50 \%$ indicated heterogeneity between studies.

\section{Results}

In this case-control study, we examined the impact of C1236T MDR1 gene polymorphism on the risk of developing AML in 131 patients with AML and 136 healthy controls. The genotypic distribution of C1236T $M D R 1$ gene polymorphism did not deviate from Hardy-Weinberg Equilibrium in controls and patients (Table 2). The clinical features of AML patients are summarized in Table 1. Mean age and sex distribution were comparable between patients and controls. When considering the FAB subtype classification, we found that $32.1 \%$ of patients had M2 and $1.1 \%$ had M7. As shown in Table 2, the genotypic frequencies were $38.9 \%$ CC, $45.8 \%$ CT, and $15.3 \%$ TT for AML patients $45.18 \%$ CC, $47.41 \%$ CT and $7.41 \%$ TT for controls, respectively. The allelic frequencies among patients were $61.83 \% \mathrm{C}, 38.17 \% \mathrm{~T}$ and $68.75 \% \mathrm{C}, 31.25 \% \mathrm{~T}$ in controls. In our study, the homozygous mutant genotype TT was statistically associated with the development of AML compared to the homozygous wild-type genotype CC (OR 2.39; 95\% CI $1.02-5.57, \mathrm{p}=0.04)$. The same trend was observed in the recessive model where we noted that carriers of 1236TT mutant genotype were more exposed to develop AML when compared to the combined $1236 \mathrm{CC} / \mathrm{CT}$ genotype (OR: 2.27, CI: 1.01-5.05, $\mathrm{p}=0.04$ ). In contrast, the dominant model did not influence the risk of the risk of AML $(\mathrm{P}>0.05)$.

Table 3 shows the distribution of clinical features of AML patients, according to the genotypes of MDR1 $C 1236 T$ polymorphism. Our results suggest that there is no significant association between this polymorphism and age at diagnosis, sex, karyotype, FAB subtypes $(\mathrm{P}>0.05)$.

Based on the studies published on MDR1 C1236T, we observed no statistically significant association between this polymorphism and AML under different genetic models $(\mathrm{P}>0.05)$. No heterogeneity between studies was found, $\mathrm{I}^{2}=0 \%$ Table 4 . 
DOI:10.31557/APJCP.2020.21.7.1899

MDR1 C1236T and Acute Myeloid Leukemia Risk

Table 1. Demographic and Clinical Parameters of AML Patients and Control Subjects

\begin{tabular}{|c|c|c|}
\hline & Cases $(\mathrm{N}=131)$ & Controls $(\mathrm{N}=136)$ \\
\hline \multicolumn{3}{|l|}{ Sex N (\%) } \\
\hline Female & $69(52.7)$ & $76(55.88)$ \\
\hline Male & $62(47.3)$ & $60(44.12)$ \\
\hline Sex N (\%) & 0.89 & 0.61 \\
\hline Age years $($ Mean \pm SD) & $37.86 \pm 15.00$ & $38.00 \pm 12.94$ \\
\hline Range & $18-74$ & $18-75$ \\
\hline \multicolumn{3}{|l|}{ FAB classification $\mathrm{N}(\%)$} \\
\hline M0 & $2(1.5)$ & \\
\hline M1 & $27(20.6)$ & \\
\hline M2 & $42(32.1)$ & \\
\hline M3 & $7(5.3)$ & \\
\hline M4 & $16(12.2)$ & \\
\hline M5 & $11(8.4)$ & \\
\hline M6 & $7(5.3)$ & \\
\hline M7 & $2(1.1)$ & \\
\hline Other & $17(13)$ & \\
\hline Median medullary blasts (\%) & $83 \%$ & \\
\hline Range & $(23-100)$ & \\
\hline Median WBC (G/L) & 19.7 & \\
\hline Range & $(0.7-560.0)$ & \\
\hline Median platelets $(\mathrm{G} / \mathrm{L})$ & 35 & \\
\hline Range & $(1-2355)$ & \\
\hline Median hemoglobin $(\mathrm{g} / \mathrm{dL})$ & 7.1 & \\
\hline Range & $(2.50-12.80)$ & \\
\hline \multicolumn{3}{|l|}{ Karyotype N (\%) } \\
\hline Normal & $48(36.6)$ & \\
\hline Abnormal & $77(58.8)$ & \\
\hline Missing & $6(4.6)$ & \\
\hline \multicolumn{3}{|l|}{ Risk group } \\
\hline Favorable & $23(17.6)$ & \\
\hline Intermediate & $71(54.2)$ & \\
\hline Adverse & $31(23.7)$ & \\
\hline Not done & $6(4.6)$ & \\
\hline
\end{tabular}

\section{Discussion}

In this case-control study, we investigated the impact of MDR1 C1236T polymorphism on acute myeloid leukemia risk in a sample of Moroccan population and
Table 2. Distribution of Genotypes and Alleles of C1236T MDR1 Gene Polymorphism in AML Patients and Controls.

\begin{tabular}{lcccc}
\hline $\begin{array}{l}\text { C1236T } \\
\text { Genotype }\end{array}$ & $\begin{array}{c}\text { Patients N } \\
(\%)\end{array}$ & $\begin{array}{c}\text { Controls N } \\
(\%)\end{array}$ & OR (95\% CI) & P-value \\
\hline CC & 131 & 136 & & \\
CT & $51(38.9)$ & $61(45.18)$ & Ref. & \\
TT & $60(45.8)$ & $65(47.41)$ & $1.10(0.66-1.84)$ & 0.7 \\
CC/CT & $20(15.3)$ & $10(7.41)$ & $2.39(1.02-5.57)$ & 0.04 \\
TT & $111(84.7)$ & $126(92.59)$ & Ref. & \\
CC & $20(15.3)$ & $10(7.41)$ & $2.27(1.01-5.05)$ & 0.04 \\
CT/TT & $51(38.9)$ & $61(45.18)$ & & \\
Allele & $80(61.1)$ & $75(54.82)$ & $1.27(0.78-2.07)$ & 0.23 \\
C & & & & \\
T & $162(61.83)$ & $187(68.75)$ & Ref & \\
HWE: $p$ value & 0.73 & 0.19 & & 0.09 \\
\hline
\end{tabular}

Table 3. Genotypic Frequencies of C1236T MDR1 Gene Polymorphism in Patients with AML According to Clinical Parameters

\begin{tabular}{lcccc}
\hline & CC & CT & TT & $P$-value \\
\hline Age of onset & & & & 0.16 \\
$\quad<20$ & 6 & 13 & 6 & \\
$>20$ & 45 & 47 & 14 & \\
Sex & & & & 0.73 \\
Female & 27 & 30 & 12 & \\
Male & 24 & 30 & 8 & \\
FAB type & & & & 0.24 \\
M0-M1 & 10 & 17 & 2 & \\
M2 & 14 & 21 & 7 & \\
M3 & 3 & 4 & 0 & \\
M4 & 7 & 6 & 3 & \\
M5 & 7 & 1 & 3 & \\
M6-M7 & 4 & 5 & 0 & \\
karyotype & & & & 0.95 \\
Normal & 18 & 22 & 8 & \\
Abnormal & 31 & 34 & 12 & \\
Risk group & & & & 0.16 \\
Favorable & 11 & 6 & 6 & \\
Intermidaite & 29 & 33 & 9 & \\
Unfavorable & 9 & 18 & 4 & \\
\hline & & & & \\
\hline
\end{tabular}

Table 4 . Pooled Analysis of Studies Exploring the Relationship between MDR1 C1236T Polymorphism and AML Risk

\begin{tabular}{|c|c|c|c|c|c|c|c|c|c|}
\hline \multirow[b]{2}{*}{ Study } & \multicolumn{3}{|c|}{ Recessive model } & \multicolumn{3}{|c|}{ Dominant model } & \multicolumn{3}{|c|}{ Allele contrast model } \\
\hline & OR & $95 \% \mathrm{CI}$ & $P$ & OR & $95 \% \mathrm{CI}$ & $P$ & OR & $95 \% \mathrm{CI}$ & $P$ \\
\hline AL-Faisal et al. 2014 [23]. & 0.81 & $0.16-3.91$ & & 2.77 & $0.59-13.05$ & & 1.39 & $0.51-3.83$ & \\
\hline Jingdong et al. 2016 [24]. & 0.91 & $0.61-1.36$ & & 0.72 & $0.39-1.34$ & & 0.88 & $1.06-1.83$ & \\
\hline Feng et al. 2016 [25]. & 0.92 & $0.62-1.37$ & & 0.89 & $0.49-1.60$ & & 0.93 & $1.13-1.97$ & \\
\hline Green et al. 2012 [26]. & 1.12 & $0.66-1.92$ & & 0.96 & $0.60-1.53$ & & 1.02 & $0.38-2.92$ & \\
\hline Total (fixed effects) & 0.95 & 0.75 to 1.22 & 0.73 & 0.91 & $0.67-1.24$ & 0.57 & 0.95 & $1.04-1.34$ & 0.59 \\
\hline Total (random effects) & 0.95 & 0.75 to 1.22 & 0.73 & 0.91 & $0.67-1.24$ & 0.58 & 0.95 & $1.00-1.39$ & 0.59 \\
\hline \multirow[t]{2}{*}{ Test for heterogeneity } & $\mathrm{Q}=0.47$ & $\mathrm{DF}=3$ & & $\mathrm{Q}=2.57$ & $\mathrm{DF}=3$ & & $\mathrm{Q}=0.98$ & $\mathrm{DF}=3$ & \\
\hline & $\mathrm{I}^{2}=0 \%$ & $\mathrm{ph}=0.92$ & & $\mathrm{I}^{2}=0 \%$ & $\mathrm{ph}=0,46$ & & $\mathrm{I}^{2}=0 \%$ & $\mathrm{ph}=0.80$ & \\
\hline
\end{tabular}




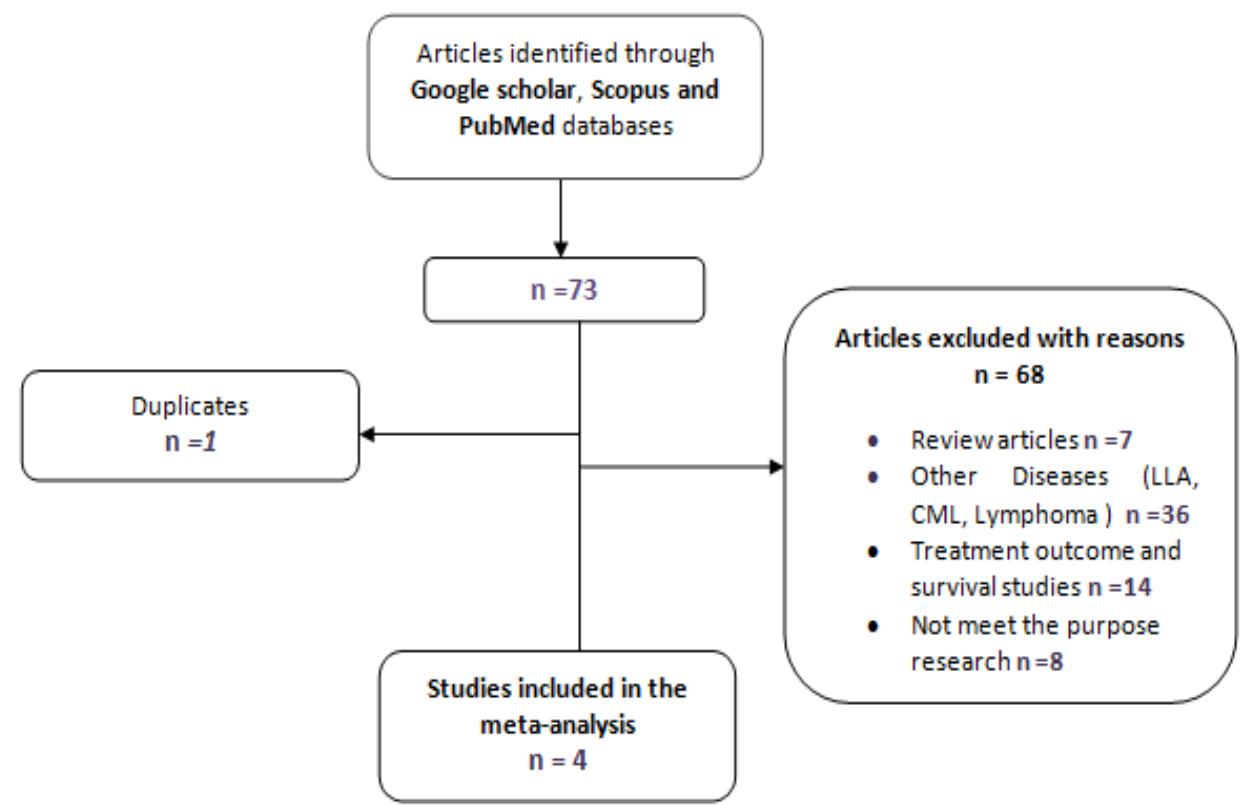

Figure 1. Flow Chart Explaining the Selection of the Included Studies in the Meta-Analysis

we found that the mutant TT genotype of MDR1 C1236T polymorphism was associated with the susceptibility of acute myeloid leukemia when compared to the wild type CC genotype. The most frequently observed FAB subtype in our study was M2; this result is consistent with our previous studies (Ait Boujmia et al., 2017; Ait Boujmia et al., 2020). Other studies have reported similar results (Habdous et al., 2004; Zendehdel et al., 2009). Regarding

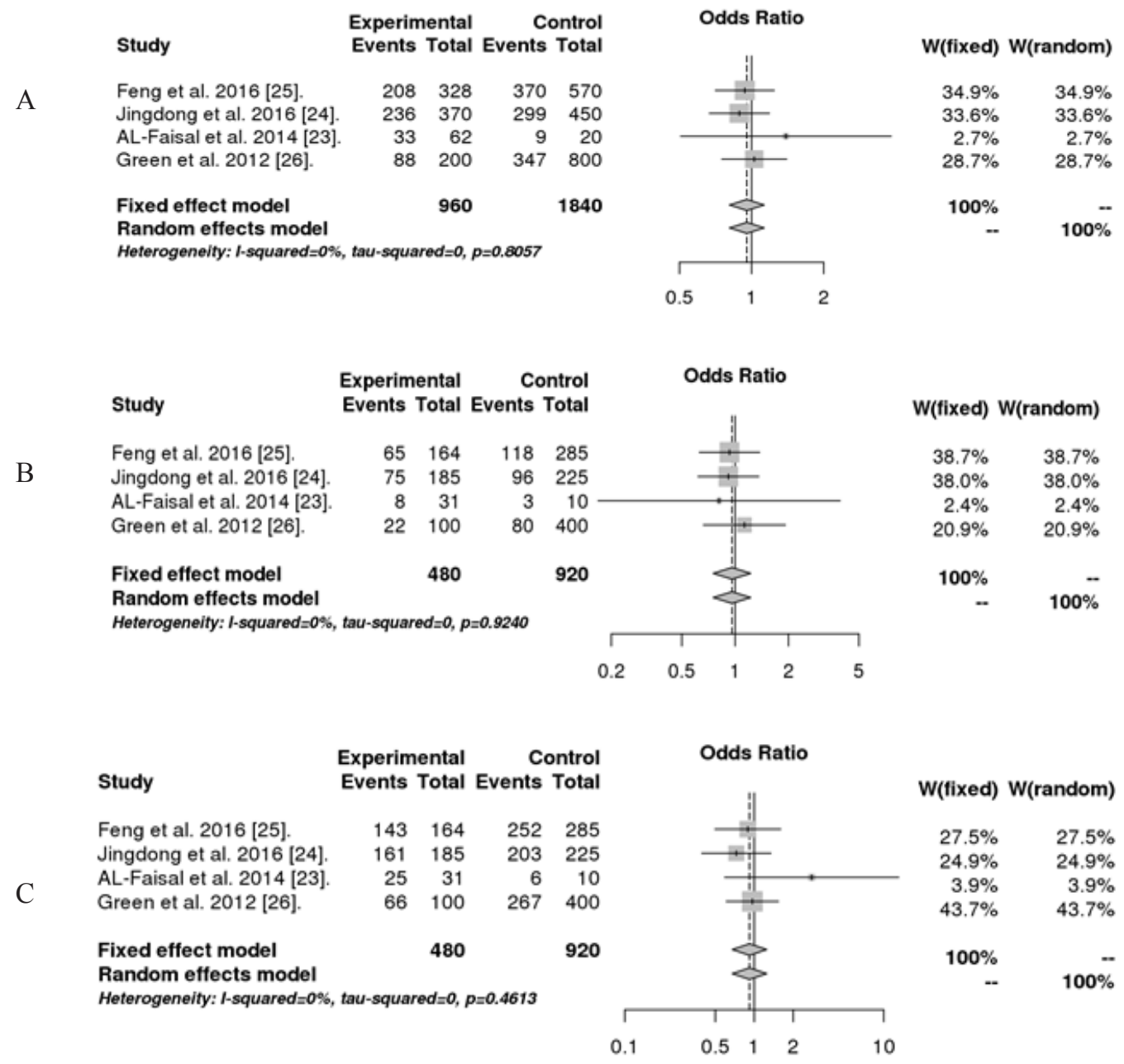

Figure 2. Forest Plots of Odds Ratios for the Association between MDR1 C1236T Polymorphism in Different Genetic Models: A, Allele contrast model; B, Recessive model; C, Dominant model. 
the correlation between this polymorphism and clinical parameters, we found that there was no statistically significant positive association between the MDR1 C1236T polymorphism and gender, age, karyotype and FAB types. Interestingly, our results showed a significant association between the TT homozygote mutant genotype and the risk of AML with an odds ratio of 2.39 (95\% CI: 1.02-5.57, $\mathrm{P}=0.04)$. AL-Faisal et al. reported a similar result in Iraqi population (Al Faisal and Al yaqubi, 2014).

In contrast, our Meta-Analysis and some studies have not shown an influence of this polymorphism and the risk of developing AML. Jingdong et al., (2016) reported that there is no significant association between MDR 1 C1236T gene polymorphism and AML risk. Feng et al., (2016) reported that no significant relationship between the ABCB1 C1236T variant and AML. Green et al., (2012) also found no significant difference in genotype frequencies between AML patients and the reference population. The difference in results between studies of MDR1 C1236T gene polymorphism could be explained by many factors such as the genetic background, the small sample size, difference in genotyping methods, and the mode of living and it should be noted that the frequency of this SNP varied according the ethnic group and to geographic location (Kassogue et al., 2013).

The influence of MDR1 C1236T gene polymorphism on the risk of AML could be explained by the intracellular accumulation of toxic and carcinogenic substances due to the low expression of MDR1 gene. Furthermore, many studies reported that the diminished MDR1 expression leads to increase DNA damage and Cytogenetic aberrations that play a critical role in the leukemogenesis (Ait Boujmia et al., 2017; Ait Boujmia et al., 2020).

In conclusion, this study results suggested that the MDR1 C1236T polymorphism could influence the risk of developing adult acute myeloid leukemia. Future studies with large sample size are necessary to confirm the association between MDR1 C1236T polymorphism and AML risk.

\section{Ethics approval / Consent Informed}

The present case-control study was approved by the Ethical Committee of Hassan II University, School of Medicine and Pharmacy, Casablanca, Morocco, Nº2/19. A written informed consent was obtained from all participants before entering the study

\section{Acknowledgments}

We are grateful to all the patients and controls who contributed to this work.

\section{Authors' contributions}

AB OK: Designed and performed experiments, performed the statistical analysis, wrote the paper.

QA: Supervised the research, discussed the study results and implications and commented on the manuscript at all stages and edited the manuscript. SN, DH, ML: Provision of study materials, reagents, materials, patients, instrumentation, revision of the manuscript.

All authors contributed extensively to the work presented in this article.

Conflicts of interest

The authors declare no conflict of interest.

\section{References}

Abuhaliema AM, Yousef AM, El-Madany NN(2016). Influence of genotype and haplotype of MDR1 (C3435T, G2677A/T, $\mathrm{C} 1236 \mathrm{~T}$ ) on the incidence of breast cancer a case-control study in Jordan. Asian Pac J Cancer Prev, 17, 261- 6.

Ait Boujmia OK, Nadifi S, Dehbi H, et al (2017). Association of Glutathione S-transferase genes (M1 and T1) with the risk of acute myeloid leukemia in a Moroccan population. Middle East J Cancer, 8, 7-12.

Ait Boujmia OK, Nadifi SD, Kassogue Y, Lamchahab M, Quessar A (2020).MDR1 gene polymorphisms and acute myeloid leukemia AML susceptibility in A Moroccan adult population: A case-control study and meta-analysi. JAN, 68, 29-35.

AL-Faisal AH, Alyaqubi KJ(2014). Effect of MDR1 Gene Expression Related with C1236T Polymorphism in Iraqi Acute Myeloid Leukemia patients. Iraqi J Biotechnol, 13, 253-65.

Ambudkar SV, Dey S, Hrycyna CA,et al (1999). Biochemical, cellular, and pharmacological aspects of the multidrug transporter. Annu Rev Pharmacol Toxicol, 39, 361-98.

Bodor M, Kelley EJ, Ho RJ (2005). Characterization of the human MDR1 gene. AAPS $J, 7,1-5$.

Clarke R, Leonessa F, Trock B (2005). Multidrug resistance/P-glycoprotein and breast cancer. review and meta-analysis. Semin Oncol, 32, 9-15.

Feng R, Zhang HX, Zhang HG, d Zhang CF (2016). Role of ABCB1 C1236T, G2677T, and C3435T genetic polymorphisms in the development of acute leukemia in a Chinese population. Genet Mol Res, 15, 10.4238/gmr.

GBD 2015 Mortality and Causes of Death Collaborators (2016). Global, regional, and national life expectancy, all-cause mortality, and cause-specific mortality for 249 causes of death, 1980-2015: a systematic analysis for the Global Burden of Disease Study 2015. Lancet, 388, 1459-1544.

Gréen H, Falk IJ, Lotfi K, et al (2012). Association of ABCB1 polymorphisms with survival and in vitro cytotoxicty in de novo acute myeloid leukemia with normal karyotype. Pharmacogenomics, 12, 111-8.

Habdous M, Siest G, Herbeth B, Vincent-Viry M, Visvikis S (2004). Glutathione S-transferases genetic polymorphisms and human diseases: overview of epidemiological studies. Ann Biol Clin, 62, 15-24.

Hattori H, Suminoe A, Morimasa W, et al (2007). Regulatory polymorphisms of multidrug resistance 1 (MDR1) gene are associated with the development of childhood acute lymphoblastic leukemia. Leuk Res, 31, 1633-40.

Hodges LM, Markova SM, Chinn LW, et al (2011). Very important pharmacogene summary: ABCB1 (MDR1, P-glycoprotein). Pharmacogenetics Genomics, 21, 152.

Hodges LM, Markova SM, Chinn LW, et al (2011). Very important pharmacogene summary: ABCB1 (MDR1, P-glycoprotein). Pharmacogenet Genomics, 21, 152-61.

Jingdong L, Cui Y, Wanling W, Xiaolin H, Ling S (2016). Association of ABCB1 C3435T and C1236T gene polymorphisms with the susceptibility to acute myeloid leukemia in a Chinese population. Int J Clin Exp Pathol, 9, 8464-70.

Kassogue Y, Dehbi H, Quachouh M, et al (2014) .Single nucleotide polymorphisms of multidrug resistance gene 1 (MDR1) and risk of chronic myeloid leukemia.Tumour

Asian Pacific Journal of Cancer Prevention, Vol $21 \quad 1903$ 
Biol, 35, 10969-75.

Kassogue Y, Dehbi H, Nassereddine S, Quachouh M, Nadifi S (2013). Genotype variability and haplotype frequency of MDR1 (ABCB1) gene polymorphism in Morocco. DNA Cell Biol, 32, 582-8.

Kim HN, Kim NY, Yu L, et al (2014). Polymorphisms in DNA repair genes and MDR1 and the risk for non-Hodgkin lymphoma. Int J Mol Sci, 15, 6703-16.

Laura M, Hodges SMM, Chinn LW, Gow JM, Kroetz DL, Klein TE, Altman RB (2011). Very important pharmacogene summary: ABCB1 (MDR1, Pglycoprotein). Pharmacogenet Genomics, 21, 152-61.

Marzolini C, Paus E, Buclin T, Kim RB (2004). Polymorphisms in human MDR1 (P-Glycoprotein): recent advances and clinical relevance. Clin Pharmacol Ther, 75, 13-33.

Miller SA, Dykes DD, Polesky HF(1988). A simple salting out procedure for extracting DNA from human nucleated cells. Nucleic Acids Res, 16, 1215.

Schinkel AH (1997). The physiological function of drug-transporting P-glycoproteins. Semin Cancer Biol, 8, 161-70.

Smith MT, Zhang L, McHale CM, Skibola CF, Rappaport SM (2011). Benzene, the exposome and future investigations of leukemia etiology. Chem Biol Interact, 192, 155-9.

Talaat RM, El-Kelliny M, El-Akhras BA, et al (2018). Association of C3435T, C1236T and C4125A polymorphisms of the MDR-1 gene in Egyptian children with acute lymphoblastic leukaemia. Asian Pac J Cancer Prev, 19, 2535-43.

Urayama KY, Wiencke JK, Buffler PA, et al (2007). MDR1 Gene variants, indoor insecticide exposure, and the risk of childhood acute lymphoblastic leukemia. Cancer Epidemiol Biomarkers Prev, 16, 1172-7.

Vardiman JW, Thiele J, Arber DA, et al (2009). The 2008 revision of the World Health Organization (WHO) classification of myeloid neoplasms and acute leukemia: rationale and important changes. Blood, 114, 937-51.

Zeeb H, Blettner M (1998). Adult leukaemia: what is the role of currently. known risk factors?. Radiat Environ Biophys, 36, 217-28.

Zendehdel K, Bahmanyar S, McCarthy S, et al (2009).Genetic polymorphisms of glutathione S-transferase genes GSTP1, GSTM1, and GSTT1 and risk of esophageal and gastric cardiacancers. Cancer Causes Control, 20, 2031-8.

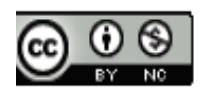

This work is licensed under a Creative Commons AttributionNon Commercial 4.0 International License. 